\title{
Effect of Different Shade House on Quality Seedling Raising of High Value Vegetables
}

\author{
A. K. M. Quamruzzaman*, Ferdouse Islam, S. R. Mallick \\ Olericulture Division, Horticulture Research Centre, Bangladesh Agricultural Research Institute, Gazipur, Bangladesh \\ Email: *akmqzs@gmail.com
}

How to cite this paper: Quamruzzaman, A.K.M., Islam, F. and Mallick, S.R. (2021) Effect of Different Shade House on Quality Seedling Raising of High Value Vegetables. American Journal of Plant Sciences, 12, 7-17.

https://doi.org/10.4236/ajps.2021.121002

Received: December 4, 2020

Accepted: January 16, 2021

Published: January 19, 2021

Copyright $\odot 2021$ by author(s) and Scientific Research Publishing Inc. This work is licensed under the Creative Commons Attribution International License (CC BY 4.0).

http://creativecommons.org/licenses/by/4.0/

\begin{abstract}
An experiment was undertaken to identify suitable shade house for different high value vegetables during the winter season of 2019-20 at the research farm of Olericulture Division, HRC, BARI, Gazipur. The experiment was randomized complete block design with 3 replications. Four vegetable varieties viz., tomato (V1), brinjal (V2), capsicum (V3), broccoli (V4) and 4 shading treatments [1) UV stabilized polyethylene film with 60 mesh insect net along with green shade net (T1); 2) UV stabilized polyethylene film with 60 mesh insect net (T2); 3) 60 mesh insect net (T3) and 4) Open field (control) (T4)] were included in this study. Different vegetables responded differently with the changes of shade house irrespective of different characters. Brinjal (V2) resulted in the maximum seed germination $(93.58 \%)$ with the lowest mortality $(5.58 \%)$, in case of shading treatment, UV stabilized polyethylene film with 60 mesh insect net along with green shade net (T1) exhibited the maximum seed germination $(94.83 \%)$ with lower mortality (3.33\%). In case of combined effect, maximum seed germination (93.58\%) and the lowest mortality (2.08\%) were observed in V2T1. The tallest seedlings were produced in V3T1 at every growth stage ( 15 days, 20 days, 25 days, 30 days). The maximum no. of leaves was produced in V3T2. The minimum insect infestation was observed in V1T1, V1T2, V2T1, V2T2, V3T1, V3T2, V4T1, V4T2 (0.67\% at 15 days and 20 days).
\end{abstract}

\section{Keywords}

Seedling, Shade House, Vegetables

\section{Introduction}

Vegetables are an important source of food and nutrition. But in Bangladesh 
vegetable production is much less than the requirement if balanced diet is provided to every individual. The present production of 4.3 million tones is to be raised to 13 million tones [1]. There are different ways and means to achieve this target, e.g., bringing additional area under vegetable crops, using hybrid seeds, use of improved agro-techniques. Another potential approach is perfection and promotion of protected cultivation of vegetables [2]. During rainy summer season, the temperature and solar radiations are adverse for growing off-season vegetables: tomato, capsicum, brinjal, cauliflower, broccoli, lettuce, cucumber, squash, netted melon, water melon. Hence, during extreme conditions of weather, these high value vegetables can be well cultivated under protected cultivation structures year-round. Research results have shown that by adopting protected cultivation, productivity of high value vegetable crops can be increased by 3 to 5 times as compared to open environment. Green house, polyhouse, shade net house \& low tunnels are the different types of protected cultivation structures commonly adopted by the farmers of developed country.

Quality seedling production is a sector having recently started growing in Bangladesh. Cultivation from seedlings has many advantageous opportunities, such as earlier harvest, utilization of time, economization of land, energy, and seeds, healthy and homogenous production, and warning process for earliness. In order to benefit from these advantages, producers have tended to production not from seeds, but from seedlings, thus increasing their demands for the seedlings of various vegetables. Cultivation from seedlings is much more important in congenial production. Use of vigorous seedlings provides solutions to the worrisome problems of the producers. In order to cultivate healthy seedlings, growing condition is as important as the ecological conditions. Various plant growing and dose studies are emphasized in the conducted researches. Efforts should be escalated for establishing and expanding growing condition, in compliance with the regulations on the production of quality vegetable seedlings, as being an important criterion. It is intended with this study to examine the quality features of the seedlings of the tomatoes and peppers, being produced in different growing conditions so as to be utilized in protected production to develop quality vegetable seedlings.

Shadenet house is a framed structure made of materials such as GI pipes, angle iron, wood or bamboo. It is covered with plastics nets which are made of $100 \%$ Polyethylene thread with specialized UV treatment having different shade percentages. It provides partially controlled atmosphere and environment by reducing light intensity and effective heat during day time to crops grown under it. Hence round the year seasonal and off-season cultivation is possible. Shading nets are used in tropical and subtropical countries for vegetable production [3] [4] [5]. Shading also reduces water requirements and increases irrigation water use efficiency in vegetable crops [6]. Poly house is a framed structure having 200 micron (800 gauges) UV stabilized transparent or translucent low density polyethylene which creates greenhouse effect making microclimate favorable for 
plant growth and development. Structure is large enough to permit a person to work inside. Poly film layer placed beneath shade net (around $1 \mathrm{~m}$ below shadenet) which increases the temperature during winter \& reduces during the summer season. This structure also aims at protect crop from devastating rains. Net house concept of protected house is a concept for protecting the insect. In well framed structure made up of GI pipes covered with 60 mesh nets.

To date, there is not much work available on protected cultivation of quality vegetables seedling production in Bangladesh condition. There is an urgent need to assess the quality vegetables seedling production and suitability for different vegetables under protected cultivation structures to meet the growing demand of the vegetables. Thus, the investigation was aimed to determine the efficacy of protected cultivation compared to open field on temperature and water requirement of vegetables during winter season. Since the selected crops viz., tomato, brinjal, capsicum, and broccoli are high value vegetables in Bangladesh that is why these vegetables are selected for these types of protected house to get quality seedlings. In this investigation an attempt is made to estimate the best protected cultivation structures for growing quality vegetables seedling production in Bangladesh condition. Keeping in view, the present study was formulated to select the suitable shade house for quality seedling raising of high value vegetables in Bangladesh.

\section{Materials and Methods}

\subsection{Experimental Site}

The experiment was designed at the research field of the Olericulture Division, HRC, BARI, Gazipur during November to December, 2019. The field was at $23.9920^{\circ} \mathrm{N}$ Latitude and $90.4125^{\circ} \mathrm{E}$ Longitudes having an elevation of $8.2 \mathrm{~m}$ from sea level under agro-ecological zone (AEZ) 28 (Annon, 1995). The farm was situated in the sub-tropical climatic zone and characterized by scanty rainfall during the experimental time.

\subsection{Treatments and Raising of Plant Materials}

Four vegetables varieties viz., tomato (V1), brinjal (V2), capsicum (V3), broccoli (V4) and 4 shading treatments [1) UV stabilized polyethylene film with 60 mesh insect net along with green shade net (T1);2) UV stabilized polyethylene film with 60 mesh insect net (T2);3) 60 mesh insect net (T3) and 4) Open field (control) (T4)] were included in this study. Total 16 treatment combinations were laid out in factorial design with three replications. Four types of vegetables were sown on polypot $(12 \mathrm{~cm} \times 10 \mathrm{~cm})$ with fungicide treated seed on $15 \mathrm{No-}$ vember 2019 under four type of shading treatments. The polypots were filled up with fine sandy-loam soil and organic manure with 50:50 ratio. Different cultural operations such as irrigation, weeding, mulching etc. were done as and when needed. 


\subsection{Air Temperatures and Relative Humidity of the Experimental Area}

Average monthly maximal and minimal air temperatures during the season in different structures are shown in Table 1 \& Table 2. Table 1, while Table 3 shows the average relative humidity.

Table 1. Average monthly minimum temperature recorded in different protected cultivation structures for winter season.

\begin{tabular}{cccccc}
\hline \multirow{2}{*}{ Treatment } & \multicolumn{5}{c}{ Minimum air temperature $\left({ }^{\circ} \mathrm{F}\right)$} \\
\cline { 2 - 6 } & Nov 2019 & Dec 2019 & Jan 2020 & Feb 2020 & Mean \\
\hline $\begin{array}{c}\text { UV stabilized polyethylene film } \\
\text { with 60 mesh insect net along } \\
\text { with green shade net }\end{array}$ & 21.5 & 16 & 14.5 & 17.5 & 17.38 \\
$\begin{array}{c}\text { UV stabilized polyethylene film } \\
\text { with 60 mesh insect net }\end{array}$ & 23 & 18.5 & 16.5 & 19.5 & 19.38 \\
60 mesh insect net & 20 & 15 & 13.5 & 16 & 16.13 \\
$\quad$ Open (control) & 19 & 14 & 12.5 & 15 & 15.13 \\
\hline
\end{tabular}

Table 2. Average monthly maximum temperature recorded in different protected cultivation structures for winter season.

\begin{tabular}{cccccc}
\hline \multirow{2}{*}{ Treatment } & \multicolumn{5}{c}{ Maximum air temperature $\left({ }^{\circ} \mathrm{F}\right)$} \\
\cline { 2 - 6 } & Nov 2019 & Dec 2019 & Jan 2020 & Feb 2020 & Mean \\
\hline $\begin{array}{c}\text { UV stabilized polyethylene film } \\
\text { with 60 mesh insect net along } \\
\text { with green shade net }\end{array}$ & 31.5 & 28.5 & 27 & 30 & 29.25 \\
$\begin{array}{c}\text { UV stabilized polyethylene film } \\
\text { with 60 mesh insect net }\end{array}$ & 34 & 31 & 29 & 32 & 31.50 \\
$\quad 60$ mesh insect net & 30 & 27 & 25.5 & 28.5 & 27.75 \\
$\quad$ Open (control) & 29 & 26 & 24.4 & 27.5 & 26.73 \\
\hline
\end{tabular}

Table 3. Average monthly mean relative humidity (\%) recorded in different protected cultivation structure during winter season.

\begin{tabular}{cccccc}
\hline \multirow{2}{*}{ Treatment } & \multicolumn{5}{c}{ Monthly mean relative humidity (\%) } \\
\cline { 2 - 6 } & Nov 2019 & Dec 2019 & Jan 2020 & Feb 2020 & Mean \\
\hline $\begin{array}{c}\text { UV stabilized polyethylene film } \\
\text { with 60 mesh insect net along } \\
\text { with green shade net }\end{array}$ & 56 & 52.5 & 48.5 & 40 & 49.25 \\
$\begin{array}{c}\text { UV stabilized polyethylene film } \\
\text { with 60 mesh insect net }\end{array}$ & 55 & 52 & 47.5 & 38.5 & 48.25 \\
$\quad 60$ mesh insect net & 54.5 & 51.5 & 47 & 38 & 47.75 \\
Open (control) & 53 & 50 & 46 & 37 & 46.50 \\
\hline
\end{tabular}




\subsection{Data Recorded}

Data were collected on seed germination (\%), seedling mortality (\%), seedling height (at 15 days, 20 days, 25 days, 30 days), no. of leaves at (at 15 days, 20 days, 25 days, 30 days), insect infestation (\%) (at 15 days, 20 days, 25 days, 30 days), from 5 randomly selected seedling from each of replication.

\subsection{Statistical Analysis}

The recorded data for different characters were analyzed statistically using MSTAT-C program to find out the variation among the different genotypes by F-test. Treatment means were compared using Duncan's Multiple Range Test (DMRT) and standard error and coefficient of variation (CV \%) were also estimated for each character.

\section{Results and Discussion}

\subsection{Effect of Vegetables Crops}

The data presented in Table 4 depicted that different vegetables [tomato (V1), brinjal (V2), capsicum (V3), broccoli (V4)] showed significant effect on performance of growth development under different shading treatments [1) UV stabilized polyethylene film with 60 mesh insect net along with green shade net (T1);2) UV stabilized polyethylene film with 60 mesh insect net (T2);3) 60 mesh insect net (T3) and 4) Open field (control) (T4)].

Brinjal (V2) resulted the maximum seed germination (93.58\%) compared to tomato (91.83\%), broccoli (91.08\%), while the lowest germination was observed in capsicum $(88.33 \%)$. In case of seedling mortality during the 30 days, the lowest mortality was observed in brinjal (5.58\%), followed by tomato (6.58\%), while the highest mortality was observed in capsicum (9.08\%). It might be due to susceptible to pest and disease. The tallest seedlings were produced in capsicum at 15 days $(10.1 \mathrm{~cm}), 20$ days $(12.4 \mathrm{~cm}), 25$ days $(14.53 \mathrm{~cm}), 30$ days $(17.7 \mathrm{~cm})$ of seedling age, while the shortest seedlings were observed in brinjal at 15 days (9.4 $\mathrm{cm}), 20$ days $(11.31 \mathrm{~cm}), 25$ days $(13.83 \mathrm{~cm})$ and 30 days $(16.65 \mathrm{~cm})$. It might be due to slow growth of brinjal seedling.

The maximum leaves were produced in capsicum at 15 days (2.86), 20 days (3.96), 25 days (3.99), 30 days (4.71), while the minimum leaves were observed in broccoli at 15 days (2.46), 20 days (3.01), 25 days (3.02) and 30 days (3.36). It might be due to slow growth of leaves. The minimum insect infestation was observed in broccoli seedling at 15 days (2.00\%), 20 days (4.33\%), 25 days (6.17\%), 30 days $(49.42 \%)$ and the maximum insect infestation was observed in brinjal at 15 days (4.94\%), 20 days (6.58\%), 30 days (13.20\%), while at 25 days, it maximum in tomato $(9.17 \%)$. It might be due to prone to insect (Figure 1).

\subsection{Effect of Different Shading Treatments}

The results revealed that among the different shading treatments [1) UV stabi- 
lized polyethylene film with 60 mesh insect net along with green shade net (T1); 2) UV stabilized polyethylene film with 60 mesh insect net (T2); 3) 60 mesh insect net (T3) and 4) Open field (control) (T4)]. The shading treatment T1 resulted the maximum seed germination (94.83\%) closely followed by T2 (914.58\%), while the lowest germination was observed in control treatment T4 (84.58\%) (Figure 2). In case of seedling mortality during the 30 days, the lowest mortality was observed in T1 (3.33\%), closely followed by T2 (5.33\%), while the highest mortality was observed in T4 (12.33\%) (Figure 3). It might be due to open condition of the seedling producing area. The tallest seedlings were produced byT1 at 15 days $(11.13 \mathrm{~cm}), 20$ days $(13.21 \mathrm{~cm}), 25$ days $(15.73 \mathrm{~cm}), 30$ days $(18.68 \mathrm{~cm})$ of seedling age, while the shortest seedlings were observed in $\mathrm{T} 4$ condition at 15 days (8.28 $\mathrm{cm}), 20$ days $(10.16 \mathrm{~cm}), 25$ days $(12.43 \mathrm{~cm})$ and 30 days $(15.58 \mathrm{~cm})$. It might be due to slow growth of seedlings in open condition (Table 5).

Table 4. Effect of vegetables crops on seed germination, seedling height, seedling mortality and insect infestation.

(a)

\begin{tabular}{|c|c|c|c|c|c|c|}
\hline \multirow{2}{*}{ Crops } & \multirow{2}{*}{$\begin{array}{c}\text { Seed } \\
\text { germination } \\
(\%)\end{array}$} & \multirow{2}{*}{$\begin{array}{c}\text { Seedling } \\
\text { mortality } \\
(\%)\end{array}$} & \multicolumn{4}{|c|}{ Seedling height at } \\
\hline & & & $\begin{array}{l}15 \text { days } \\
(\mathrm{cm})\end{array}$ & $\begin{array}{c}20 \text { days } \\
(\mathrm{cm})\end{array}$ & $\begin{array}{l}25 \text { days } \\
(\mathrm{cm})\end{array}$ & $\begin{array}{l}30 \text { days } \\
(\mathrm{cm})\end{array}$ \\
\hline Tomato (V1) & $91.83 \mathrm{~b}$ & $6.58 c$ & $9.8 \mathrm{~b}$ & $11.78 \mathrm{ab}$ & $14.23 \mathrm{ab}$ & $17.4 \mathrm{a}$ \\
\hline Brinjal (V2) & $93.58 \mathrm{a}$ & $5.58 \mathrm{~d}$ & $9.4 \mathrm{c}$ & $11.31 \mathrm{~b}$ & $13.83 \mathrm{~b}$ & $16.65 \mathrm{~b}$ \\
\hline Capsicum (V3) & $88.33 c$ & $9.08 \mathrm{a}$ & $10.1 \mathrm{a}$ & $12.08 \mathrm{a}$ & $14.53 \mathrm{a}$ & $17.7 \mathrm{a}$ \\
\hline Broccoli (V4) & $91.08 \mathrm{~b}$ & $7.33 \mathrm{~b}$ & $9.6 \mathrm{bc}$ & $11.51 \mathrm{~b}$ & $14.03 \mathrm{~b}$ & $16.85 \mathrm{~b}$ \\
\hline Level of sig. & $* * *$ & $* * *$ & $* * *$ & * & $* *$ & $* * *$ \\
\hline (CV\%) & 1.85 & 8.21 & 3.62 & 5.40 & 3.42 & 2.82 \\
\hline
\end{tabular}

Level of sig. $={ }^{\star * *}=0.1 \% ;{ }^{\star *}=1 \% ;{ }^{\star}=5 \%$.

(b)

\begin{tabular}{|c|c|c|c|c|}
\hline \multirow{2}{*}{ Crops } & \multicolumn{4}{|c|}{ No. of leaves at } \\
\hline & 15 days & 20 days & 25 days & 30 days \\
\hline Tomato (V1) & $2.56 \mathrm{~b}$ & $3.66 \mathrm{~b}$ & $3.69 \mathrm{~b}$ & $4.41 \mathrm{~b}$ \\
\hline Brinjal (V2) & $2.56 \mathrm{~b}$ & $3.66 \mathrm{~b}$ & $3.69 \mathrm{~b}$ & $4.41 \mathrm{~b}$ \\
\hline Capsicum (V3) & $2.86 \mathrm{a}$ & $3.96 \mathrm{a}$ & $3.99 \mathrm{a}$ & $4.71 \mathrm{a}$ \\
\hline Broccoli (V4) & $2.46 \mathrm{~b}$ & $3.01 \mathrm{c}$ & $3.02 \mathrm{c}$ & $3.36 \mathrm{c}$ \\
\hline Level of significance & $* * *$ & $* * *$ & $* * *$ & $* * *$ \\
\hline$(\mathrm{CV} \%)$ & 7.87 & 0.82 & 6.52 & 4.87 \\
\hline
\end{tabular}

Level of sig. $=^{\star * *}=0.1 \% ;{ }^{* *}=1 \% ;^{*}=5 \%$; CV: coefficient of variation. 


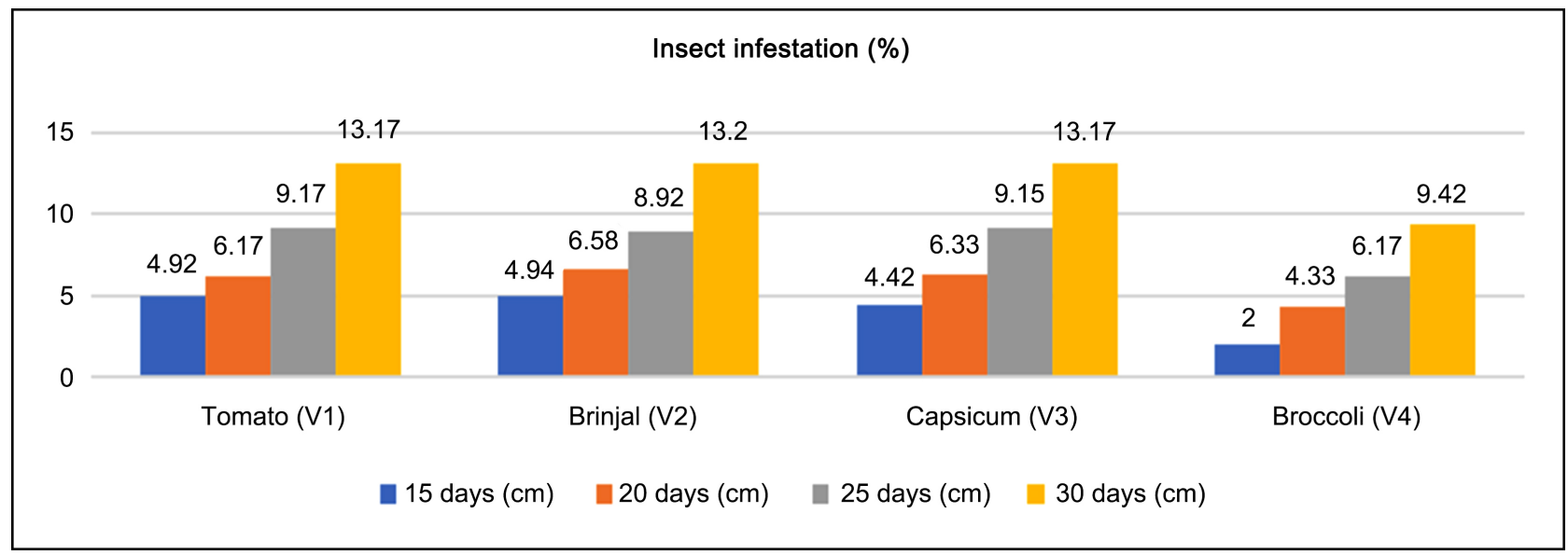

Figure 1. Effect of vegetables crops on insect infestation (\%).

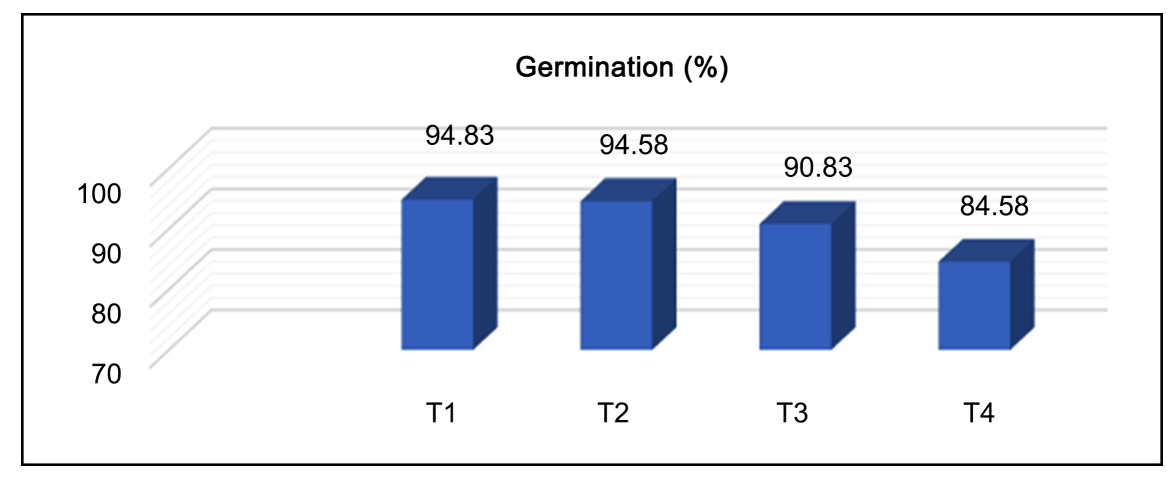

Figure 2. Effect of different shading treatments on seed germination.

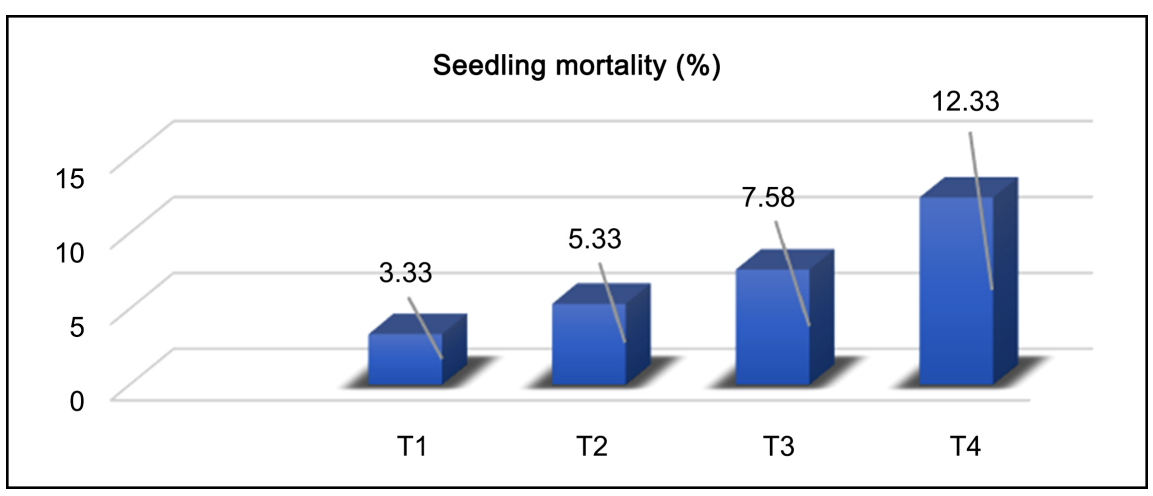

Figure 3. Effect of different shading treatments on seedling mortality.

The maximum no. of leaves was produced in T2 at 15 days (3.08), 20 days (3.93), 25 days (3.89), 30 days (4.81), while the minimum no. of leaves were observed in T4 at 15 days (2.08), 20 days (3.11), 25 days (3.03) and 30 days (3.81). It might be due to less production of leaves. The minimum insect infestation was observed in $\mathrm{T} 1$ at 15 days (0.65\%), 20 days (1.17\%) and in $\mathrm{T} 2$ it was observed at 25 days $(2.42 \%), 30$ days $(4.92 \%)$ and the maximum insect infestation was observed in T4 at 15 days (13.83\%), 20 days (19.33\%), 30 days (23.63\%), 25 days (31.92\%). It might be due to open condition which favors insect for infestation. 
Table 5. Effect of different shading treatments on seed germination, seedling mortality, seedling height and insect infestation.

(a)

\begin{tabular}{ccccc}
\hline \multirow{2}{*}{ Crops } & \multicolumn{4}{c}{ Seedling height at } \\
\cline { 2 - 5 } & 15 days & 20 days & 25 days & 30 days \\
\hline T1 & $11.13 \mathrm{a}$ & $13.21 \mathrm{a}$ & $15.73 \mathrm{a}$ & $18.68 \mathrm{a}$ \\
T2 & $10.18 \mathrm{~b}$ & $12.21 \mathrm{~b}$ & $15.03 \mathrm{~b}$ & $17.73 \mathrm{~b}$ \\
T3 & $9.33 \mathrm{c}$ & $11.11 \mathrm{c}$ & $13.43 \mathrm{c}$ & $16.63 \mathrm{c}$ \\
T4 & $8.28 \mathrm{~d}$ & $10.16 \mathrm{~d}$ & $12.43 \mathrm{~d}$ & $15.58 \mathrm{~d}$ \\
Level of sig. & $* * *$ & $* * *$ & $* * *$ & $* * *$ \\
CV & 3.2 & 5.40 & 3.42 & 2.82 \\
\hline
\end{tabular}

Level of sig. $=^{* *}=0.1 \%$; ${ }^{*}=1 \%{ }^{*}=5 \%$; T1: UV stabilized polyethylene film with 60 mesh insect net along with green shade net; T2: UV stabilized polyethylene film with 60 mesh insect net; T3: 60 mesh insect net; T4: Open field (control).

(b)

\begin{tabular}{ccccccccc}
\hline & \multicolumn{3}{c}{ No. of leaves at } & \multicolumn{5}{c}{ Insect infestation (\%) } \\
\cline { 2 - 8 } Crops & 15 days & 20 days & 25 days & 30 days & 15 days & 20 days & 25 days & 30 days \\
\hline T1 & $2.88 \mathrm{~b}$ & $3.68 \mathrm{~b}$ & $3.69 \mathrm{~b}$ & $4.21 \mathrm{~b}$ & $0.65 \mathrm{~b}$ & $1.17 \mathrm{~b}$ & $2.92 \mathrm{c}$ & $5.17 \mathrm{c}$ \\
T2 & $3.08 \mathrm{a}$ & $3.93 \mathrm{a}$ & $3.89 \mathrm{a}$ & $4.81 \mathrm{a}$ & $0.67 \mathrm{~b}$ & $1.25 \mathrm{~b}$ & $2.42 \mathrm{c}$ & $4.92 \mathrm{c}$ \\
T3 & $2.38 \mathrm{c}$ & $3.56 \mathrm{c}$ & $3.49 \mathrm{c}$ & $4.06 \mathrm{~b}$ & $1.08 \mathrm{~b}$ & $1.67 \mathrm{~b}$ & $4.42 \mathrm{~b}$ & $6.92 \mathrm{~b}$ \\
T4 & $2.08 \mathrm{~d}$ & $3.11 \mathrm{~d}$ & $3.32 \mathrm{c}$ & $3.81 \mathrm{c}$ & $13.83 \mathrm{a}$ & $19.33 \mathrm{a}$ & $23.67 \mathrm{a}$ & $31.92 \mathrm{a}$ \\
Level of sig. & $* * *$ & $* * *$ & $* * *$ & $* * *$ & $* * *$ & $* * *$ & $* * *$ & $* * *$ \\
CV (\%) & 7.87 & 0.82 & 6.52 & 4.87 & 18.08 & 17.16 & 12.26 & 8.38 \\
\hline
\end{tabular}

Level of sig. ${ }^{* * *}=0.1 \%$; ${ }^{*}=1 \%$; ${ }^{*}=5 \%$; T1: UV stabilized polyethylene film with 60 mesh insect net along with green shade net; T2: UV stabilized polyethylene film with 60 mesh insect net; T3: 60 mesh insect net; T4: Open field (control).

\subsection{Effect of Interaction of Different Vegetables and Shading Treatments}

The maximum seed germination (93.58\%) was observed in V2T1 compared to V1T2, V4T1 (95.83\%), while the lowest germination was observed in V3T4 $(81.83 \%)$. In case of seedling mortality during the 30 days, lowest mortality was observed in V2T1 (2.08\%), followed by V1T1, V4T1 (3.08\%), while the highest mortality was observed in V3T4 (15.08\%). It might be due to susceptible to pest and disease. The tallest seedlings were produced in V3T1 at 15 days $(11.50 \mathrm{~cm})$, 20 days $(13.73 \mathrm{~cm}), 25$ days $(16.15 \mathrm{~cm}), 30$ days $(19.25 \mathrm{~cm})$ followed by V1T1 at 15 days $(11.20 \mathrm{~cm}), 20$ days $(13.43 \mathrm{~cm}), 25$ days $(15.85 \mathrm{~cm}), 30$ days $(18.95 \mathrm{~cm})$ while the shortest seedlings were observed in V2T4 at 15 days $(8.00 \mathrm{~cm}), 20$ days $(9.93 \mathrm{~cm}), 25$ days $(12.15 \mathrm{~cm})$ and 30 days $(15.15 \mathrm{~cm})$. Seedling growth was slow due to open condition (Table 6). 
Table 6. Interaction effect of different vegetables and shading treatments.

(a)

\begin{tabular}{ccccccc}
\hline \multirow{2}{*}{ Crops } & Germination & Seedling mortality & \multicolumn{4}{c}{ Seedling height at } \\
\cline { 4 - 7 } & $(\%)$ & $(\%)$ & 15 days & 20 days & 25 days & 30 days \\
\hline V1T1 & $94.83 \mathrm{a}-\mathrm{c}$ & $3.08 \mathrm{i}$ & $11.20 \mathrm{ab}$ & $13.43 \mathrm{ab}$ & $15.85 \mathrm{ab}$ & $18.95 \mathrm{ab}$ \\
V1T2 & $95.83 \mathrm{ab}$ & $5.08 \mathrm{~g}$ & $10.30 \mathrm{de}$ & $12.33 \mathrm{c}-\mathrm{e}$ & $15.15 \mathrm{~b}-\mathrm{d}$ & $18.05 \mathrm{~cd}$ \\
V1T3 & $91.83 \mathrm{def}$ & $6.08 \mathrm{f}$ & $9.40 \mathrm{~g}-\mathrm{i}$ & $11.23 \mathrm{f}-\mathrm{i}$ & $13.45 \mathrm{ef}$ & $16.85 \mathrm{e}-\mathrm{g}$ \\
V1T4 & $84.83 \mathrm{~h}$ & $12.08 \mathrm{~b}$ & $8.30 \mathrm{kl}$ & $10.13 \mathrm{j}$ & $12.45 \mathrm{gh}$ & $15.75 \mathrm{~h}-\mathrm{j}$ \\
V2T1 & $95.83 \mathrm{ab}$ & $2.08 \mathrm{j}$ & $10.80 \mathrm{~b}-\mathrm{d}$ & $12.73 \mathrm{a}-\mathrm{d}$ & $15.35 \mathrm{a}-\mathrm{d}$ & $18.15 \mathrm{~b}-\mathrm{d}$ \\
V2T2 & $96.83 \mathrm{a}$ & $4.08 \mathrm{~h}$ & $9.80 \mathrm{e}-\mathrm{g}$ & $11.83 \mathrm{~d}-\mathrm{g}$ & $14.65 \mathrm{~d}$ & $17.15 \mathrm{ef}$ \\
V2T3 & $93.83 \mathrm{~b}-\mathrm{d}$ & $6.08 \mathrm{f}$ & $9.00 \mathrm{ij}$ & $10.73 \mathrm{~h}-\mathrm{j}$ & $13.15 \mathrm{e}-\mathrm{g}$ & $16.15 \mathrm{~g}-\mathrm{i}$ \\
V2T4 & $87.83 \mathrm{~g}$ & $10.08 \mathrm{c}$ & $8.00 \mathrm{l}$ & $9.93 \mathrm{j}$ & $12.15 \mathrm{~h}$ & $15.15 \mathrm{j}$ \\
V3T1 & $92.83 \mathrm{c}-\mathrm{e}$ & $5.08 \mathrm{~g}$ & $11.50 \mathrm{a}$ & $13.73 \mathrm{a}$ & $16.15 \mathrm{a}$ & $19.25 \mathrm{a}$ \\
V3T2 & $90.83 \mathrm{ef}$ & $7.08 \mathrm{e}$ & $10.60 \mathrm{~cd}$ & $12.63 \mathrm{~b}-\mathrm{d}$ & $15.45 \mathrm{a}-\mathrm{d}$ & $18.33 \mathrm{bc}$ \\
V3T3 & $87.83 \mathrm{~g}$ & $9.08 \mathrm{~d}$ & $9.70 \mathrm{f}-\mathrm{h}$ & $11.53 \mathrm{e}-\mathrm{h}$ & $13.75 \mathrm{e}$ & $17.15 \mathrm{ef}$ \\
V3T4 & $81.83 \mathrm{i}$ & $15.08 \mathrm{a}$ & $8.60 \mathrm{jk}$ & $10.43 \mathrm{ij}$ & $12.75 \mathrm{f}-\mathrm{h}$ & $16.05 \mathrm{~g}-\mathrm{i}$ \\
V4T1 & $95.83 \mathrm{ab}$ & $3.08 \mathrm{i}$ & $11.00 \mathrm{a}-\mathrm{c}$ & $12.93 \mathrm{a}-\mathrm{c}$ & $15.55 \mathrm{a}-\mathrm{c}$ & $18.35 \mathrm{bc}$ \\
V4T2 & $94.83 \mathrm{a}-\mathrm{c}$ & $5.08 \mathrm{~g}$ & $10.00 \mathrm{ef}$ & $12.03 \mathrm{c}-\mathrm{f}$ & $14.85 \mathrm{~cd}$ & $17.35 \mathrm{de}$ \\
V4T3 & $89.83 \mathrm{fg}$ & $9.08 \mathrm{~d}$ & $9.20 \mathrm{hi}$ & $10.93 \mathrm{~g}-\mathrm{j}$ & $13.35 \mathrm{ef}$ & $16.35 \mathrm{f}-\mathrm{h}$ \\
V4T4 & $83.83 \mathrm{hi}$ & $12.08 \mathrm{~b}$ & $8.20 \mathrm{kl}$ & $10.13 \mathrm{j}$ & $12.35 \mathrm{gh}$ & $-15.35 \mathrm{ij}$
\end{tabular}

Level of sig. $=^{* *}=0.1 \% ;{ }^{* *}=1 \%{ }^{*}=5 \%$; V1: Tomato, V2: brinjal, V3: capsicum, V4: broccoli; T1: UV stabilized polyethylene film with 60 mesh insect net along with green shade net; T2: UV stabilized polyethylene film with 60 mesh insect net; T3: 60 mesh insect net; T4: Open field (control).

(b)

\begin{tabular}{|c|c|c|c|c|c|c|c|c|}
\hline \multirow{2}{*}{ Crops } & \multicolumn{4}{|c|}{ No. of leaves at } & \multicolumn{4}{|c|}{ Insect infestation (\%) } \\
\hline & 15 days & 20 days & 25 days & 30 days & 15 days & 20 days & 25 days & 30 days \\
\hline V1T1 & $2.83 \mathrm{~b}-\mathrm{d}$ & $3.73 \mathrm{~d}$ & $3.77 \mathrm{~b}-\mathrm{d}$ & $4.33 \mathrm{~b}-\mathrm{d}$ & $0.67 \mathrm{~d}$ & $0.67 \mathrm{~d}$ & $3.17 \mathrm{ef}$ & $5.17 \mathrm{de}$ \\
\hline V1T2 & $3.03 \mathrm{a}-\mathrm{c}$ & $4.00 \mathrm{~b}$ & $3.97 \mathrm{ab}$ & $5.03 \mathrm{a}$ & $0.67 \mathrm{~d}$ & $0.67 \mathrm{~d}$ & $2.17 \mathrm{f}$ & $5.17 \mathrm{de}$ \\
\hline V1T3 & $2.33 \mathrm{ef}$ & $3.63 \mathrm{e}$ & $3.57 c-e$ & $4.23 \mathrm{~cd}$ & $1.00 \mathrm{~d}$ & $1.00 \mathrm{~d}$ & $4.17 \mathrm{de}$ & $7.17 \mathrm{c}$ \\
\hline V1T4 & $2.03 \mathrm{fg}$ & $3.23 \mathrm{~h}$ & $3.47 \mathrm{~d}-\mathrm{f}$ & $4.03 \mathrm{de}$ & $17.30 \mathrm{a}$ & $22.30 \mathrm{a}$ & $27.15 \mathrm{a}$ & $35.15 \mathrm{a}$ \\
\hline $\mathrm{V} 2 \mathrm{~T} 1$ & $2.83 \mathrm{~b}-\mathrm{d}$ & $3.73 \mathrm{~d}$ & $3.77 \mathrm{~b}-\mathrm{d}$ & $4.33 \mathrm{~b}-\mathrm{d}$ & $0.67 \mathrm{~d}$ & $1.33 \mathrm{~d}$ & $2.17 \mathrm{f}$ & $5.17 \mathrm{de}$ \\
\hline $\mathrm{V} 2 \mathrm{~T} 2$ & $3.03 \mathrm{a}-\mathrm{c}$ & $4.01 \mathrm{~b}$ & $3.97 \mathrm{ab}$ & $5.03 \mathrm{a}$ & $0.67 \mathrm{~d}$ & $2.33 \mathrm{~d}$ & $3.17 \mathrm{ef}$ & $5.17 \mathrm{de}$ \\
\hline V2T3 & $2.33 \mathrm{ef}$ & $3.63 \mathrm{e}$ & $3.57 \mathrm{c}-\mathrm{e}$ & $4.23 \mathrm{~cd}$ & $1.00 \mathrm{~d}$ & $2.33 \mathrm{~d}$ & $5.17 \mathrm{~d}$ & $7.17 \mathrm{c}$ \\
\hline $\mathrm{V} 2 \mathrm{~T} 4$ & $2.03 \mathrm{fg}$ & $3.23 \mathrm{~h}$ & $3.47 \mathrm{~d}-\mathrm{f}$ & $4.03 \mathrm{de}$ & $15.33 \mathrm{~b}$ & $20.33 \mathrm{~b}$ & $25.17 \mathrm{~b}$ & $35.17 \mathrm{a}$ \\
\hline V3T1 & $3.13 \mathrm{ab}$ & $4.03 \mathrm{~b}$ & $4.07 \mathrm{ab}$ & $4.63 \mathrm{~b}$ & $0.67 \mathrm{~d}$ & $1.33 \mathrm{~d}$ & $3.17 \mathrm{ef}$ & $5.17 \mathrm{de}$ \\
\hline V3T2 & $3.33 \mathrm{a}$ & $4.33 \mathrm{a}$ & $4.27 \mathrm{a}$ & $5.33 \mathrm{a}$ & $0.67 \mathrm{~d}$ & $0.67 \mathrm{~d}$ & $2.17 \mathrm{f}$ & $5.17 \mathrm{de}$ \\
\hline V3T3 & $2.63 \mathrm{de}$ & $3.93 \mathrm{c}$ & $3.87 \mathrm{bc}$ & $4.53 \mathrm{bc}$ & $1.00 \mathrm{~d}$ & $1.00 \mathrm{~d}$ & $4.17 \mathrm{de}$ & $7.17 \mathrm{c}$ \\
\hline V3T4 & $2.33 \mathrm{ef}$ & $3.53 \mathrm{f}$ & $3.77 \mathrm{~b}-\mathrm{d}$ & $4.33 \mathrm{dcd}$ & $17.33 \mathrm{a}$ & $22.33 \mathrm{a}$ & $27.17 \mathrm{a}$ & $35.17 \mathrm{a}$ \\
\hline V4T1 & $2.73 \mathrm{~cd}$ & $3.23 \mathrm{~h}$ & $3.17 \mathrm{fg}$ & $3.53 \mathrm{fg}$ & $0.67 \mathrm{~d}$ & $1.33 \mathrm{~d}$ & $3.17 \mathrm{ef}$ & $5.17 \mathrm{de}$ \\
\hline V4T2 & $2.93 \mathrm{~b}-\mathrm{d}$ & $3.33 \mathrm{~g}$ & $3.37 \mathrm{ef}$ & $3.83 \mathrm{ef}$ & $0.67 \mathrm{~d}$ & $1.33 \mathrm{~d}$ & $2.17 \mathrm{f}$ & $4.17 \mathrm{e}$ \\
\hline V4T3 & $2.23 \mathrm{fg}$ & $3.03 \mathrm{i}$ & $2.97 \mathrm{~g}$ & $3.23 \mathrm{~g}$ & $1.33 \mathrm{~d}$ & $2.33 \mathrm{~d}$ & $4.17 \mathrm{de}$ & $6.17 \mathrm{~cd}$ \\
\hline V4T4 & $1.93 \mathrm{~g}$ & $2.43 \mathrm{j}$ & $2.57 \mathrm{~h}$ & $2.83 \mathrm{~h}$ & $5.33 c$ & $12.33 \mathrm{c}$ & $15.17 \mathrm{c}$ & $22.17 \mathrm{~b}$ \\
\hline Sig Level & - & $* * *$ & - & - & $* * *$ & $* * *$ & $* * *$ & $* * *$ \\
\hline CV (\%) & 7.87 & 0.82 & 6.52 & 4.87 & 18.08 & 17.16 & 12.26 & 8.38 \\
\hline
\end{tabular}

Level of sig. $=^{* *}=0.1 \%$; ${ }^{* *}=1 \%{ }^{*}=5 \%$; V1: Tomato, V2: brinjal, V3: capsicum, V4: broccoli; T1: UV stabilized polyethylene film with 60 mesh insect net along with green shade net; T2: UV stabilized polyethylene film with 60 mesh insect net; T3: 60 mesh insect net; T4: Open field (control). 
The maximum no. of leaves was produced in V3T2 at 15 days (33.33), 20 days (4.33), 25 days (4.27), 30 days (5.33), followed by V3T1 at 15 days (3.13), 20 days (4.03), 25 days (4.07), 30 days (4.63), while the minimum no. of leaves were observed in V4T4 at 15 days (1.93), 20 days (2.43), 25 days (2.57) and 30 days (2.83). It might be due to less production of leaves at open condition. The minimum insect infestation was observed in V1T1, V1T2, V2T1, V2T2, V3T1, V3T2, V4T1, V4T2 (0.67\% at 15 days and 20 days), while $2.17 \%$ was at 25 days and $4.17 \%$ at 30 days and the maximum insect infestation was observed in V3T4 at 15 days (17.33\%), 20 days (22.33\%), 30 days (27.17\%), 25 days (35.17\%). It might be due to open condition which favors insect for infestation.

\section{Conclusion}

Brinjal (V2) resulted in the maximum seed germination (93.58\%) with the lowest mortality (5.58\%), in case of shading treatment, UV stabilized polyethylene film with 60 mesh insect net along with green shade net (T1) exhibited the maximum seed germination (94.83\%) with lower mortality (3.33\%). In case of combined effect, maximum seed germination (93.58\%) and the lowest mortality $(2.08 \%)$ were observed in V2T1. The tallest seedlings were produced in V3T1 at every growth stage (15 days, 20 days, 25 days, 30 days). The maximum no. of leaves was produced in V3T2. The minimum insect infestation was observed in V1T1, V1T2, V2T1, V2T2, V3T1, V3T2, V4T1, V4T2 (0.67\% at 15 days and 20 days).

\section{Acknowledgements}

This work was supported by the project "Development of protective culture technology for safe and quality vegetables and fruit production", funded by PIU-BARC, NATP-2 to complete this work.

\section{Conflicts of Interest}

The authors declare no conflicts of interest regarding the publication of this paper.

\section{References}

[1] Anonymous (2020) Year Book of Agricultural Statistics of Bangladesh 2018. Bangladesh Bureau of Statistics, Ministry of Planning, Government of Peoples Republic of Bangladesh, Dhaka, Bangladesh, 249-290.

[2] Sanwal, S.K. Patel, K.K. and Yadav, D.S. (2004) Vegetable Production under Protected Conditions in NEH Region: Problems and Prospects. ENVIS Bulletin: Himalayan Ecology, 12.

[3] Castellano, S., Mugnozza, G.S., Russo, G., Briassoulis, D., Mistriotis, A., Hemming, S. and Waaijenberg, D. (2008) Plastics Net in Agriculture: A General Review of Types and Applications. Applied Engineering in Agriculture, 24, 799-808. https://doi.org/10.13031/2013.25368

[4] Ilic, Z.S., Milenkovic, L., Stanojevic, L., Cvetkovic, D. and Fallik, E. (2012) Effects of 
the Modification of Light Intensity by Color Shade Nets on Yield and Quality of Tomato Fruits. Scientia Horticulturae, 139, 90-95.

https://doi.org/10.1016/j.scienta.2012.03.009

[5] Kittas, C., Katsoulas, N., Rigakis, N., Bartzanas, T. and Kitta, E. (2012) Effects on Microclimate, Crop Production and Quality of a Tomato Crop Grown under Shade Nets. The Journal of Horticultural Science and Biotechnology, 87, 7-12. https://doi.org/10.1080/14620316.2012.11512822

[6] Moller, M. and Assouline, S. (2007) Effects of a Shading Screen on Microclimate and Crop Water Requirements. Irrigation Science, 25, 171-181.

https://doi.org/10.1007/s00271-006-0045-9 